\title{
THE PENNING DISCHARGE EXPERIMENTAL STUDY AND ITS SIMULATION.
}

\author{
Mamedov N. V.*, Schitov N. N., Lobok M. G., Kanshin I. A. \\ All-Russia Research Institute of Automatics (VNIIA), Moscow, 127055, Russia \\ * m_nikitos@mail.ru
}

\begin{abstract}
The influence of the demountable Penning ion source electrodes geometry on the discharge characteristics and extracted ion current is investigated. The extracted currents, ignition potentials and the operational pressure ranges are compared at different anode heights and its arrangement relative to the discharge cell. The use of ring and mesh anodes has allowed to visualize the discharge burning areas and their structure versus the pressure, anode potential and cell geometry. Some PIC simulations of Penning discharge are made in the Vorpal code. The good correspondence between the simulated electron density and plasma glow areas on the photos is shown. The other one-particle simulation has shown that the magnetic field nonuniformity significantly affects the ignition and combustion discharge potentials.
\end{abstract}

Keywords: Penning discharge, Penning source, current-voltage characteristic, PIC simulation.

\section{Introduction}

The oscillating electrons discharge in crossed fields (Penning discharge) is used into different devices, particularly ion sources (PIG-sources) [1, [2, [3], [4]. Regardless of the specific purpose the optimization problem of geometrical as well as physical parameters of the discharge cell insuring reliable and effective discharge combustion and charged particles extraction is extremely important. No less important is the establishment of some general, fundamental laws, similarity criteria allowing the extrapolation of the concrete devices investigation results onto the whole class of similar devices.

A lot of Penning cells differing by their geometrical parameters, as well as other characteristics - the anode potential, magnetic field magnitude and configuration and so far are described. That is why the investigation results of concrete ion sources (IS) prototypes are often hardly compared. Likewise different theoretical discharge models have different domains of applicability and generality degree. The separate question is the influence of magnetic field uniformity. There are no grounds to state that the investigation results obtained in the ultimate uniform field (e.g. long solenoid) may be extrapolated on the constant magnets nonuniform magnetic field.

Hence the discrepant experimental data and different theoretical appreciations spur to explore the discharge in the united experiment - physical (fullscale) and digital.

\section{The physical and computer experiments description.}

The physical experiment was carried on the specially designed test bench with the pumping system TPScompact (turbo-molecular pump TV81M and spiral pump IDP-3) Varian. The demountable IS was installed trough dielectric spacers on the conductor studbolts, that is why one was able to displace the IS electrodes together as well as separately along the cell axe with the help of rotary motion feedthroughs. The electrodes had different design and were made of different materials. The discharge and extracted currents, ignition potentials and operational pressure ranges at different cell configurations were measured during the experiments Fig. 1 The energy efficiency was appreciated as the ratio of the extracted current to the discharge power consumption.

The ring anode $\left(\mathrm{D}_{a} / \mathrm{h}_{a}\right.$ «1) was made of molybdenum wire $(0.5 \mathrm{~mm}$ diameter $)$, the short $\left(\mathrm{D}_{a} / \mathrm{h}_{a}=2\right)$ and long $\left.\mathrm{D}_{a} / \mathrm{h}_{a}=1\right)$ anodes-cylinders of stainless steel wire mesh (1x1 mm cell). The cathode was made of aluminum $\left(\mathrm{D}_{c}<\mathrm{D}_{a}\right)$, the anti-cathode $\left(\mathrm{D}_{c}=\mathrm{D}_{a c}\right)$ was made of nickel mesh $(2 \times 2 \mathrm{~mm}$ cell $)$ to eliminate the finite extraction aperture area influence. The extracted current was measured with the Faraday Cup $\left(\mathrm{D}_{F C} \sim \mathrm{D}_{a}\right)$ equipped with the magnetic system of secondary electrons suppression. To visualize the discharge combustion areas the Penning magnetic system corresponded to the assemblies of some ring magnets, mounted behind the cathode and anti-cathode [5]. The high purity (99.999\%) hydrogen was chosen as a working gas. The measurements automation (Labview code) allowed to take of current - voltage characteristics of the discharge and extracted ion current together with the video - registration of the discharge combustion inside the IS. The glow areas on photos were processed with ImageJ code.

Experimental and estimation data of the concrete cell geometry were compared with the PIC simulation results obtained with the program code Vorpal [6] (see Fig. 2). The description of the charged particle interaction was divided into two stages: the calculation of 

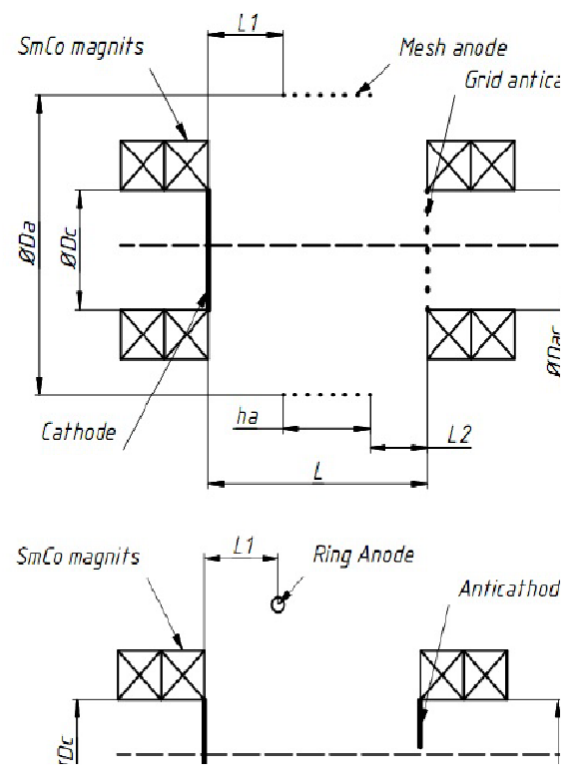

Figure 1. The schematic drawing of the ion source: $h_{a}$ - an anode height; $D_{a}$ - an anode diameter; $L_{1}$ a cathode-anode distance; $L_{2}$ - a anode-anticathode distance; $D_{c}$ and $D_{a c}$ - cathode and anticathode diameters; $L$ - a discharge cell length (cathode-anticathode distance)

the fields created by the particles, and the determination particles motion. The electric field analysis was carried out using the Poisson equation; the determination of the charged particles motion was carried out by the Boris method [7. The magnetic field was set in accordance with the measured setuo on-axis data (see fig 3). The simulation model was supplemented with kinetic calculations of the surrounding gas (hydrogen molecules) ionization and taken into account the electrons scattering by Monte Carlo method. The task was complemented by the boundary conditions of the first kind on the boundary of the computational domain and on the surface of conductors. The resulting system of differential equations was solved by iterative method of generalized minimum residuals 8. The IS plasma parameters were computed using the electron elastic scattering and hydrogen molecule cross-sections. The energy spectrum and angular distribution of the secondary ion-electron emission from cathode and anti-cathode were calculated taking into account data from [9]. The secondary ion-electron emission coefficient was chosen equal to 0.3 . The computation area was from 2.097 to 4.096 million of cells. Under this conditions the current-voltage characteristics of the discharge and extracted currents were calculated.

\section{The magnetic field uniformity/nonuniformity influence. The one-particle estimates.}

The variable magnetic induction magnitude determined by the mutual cathodes arrangement is one of

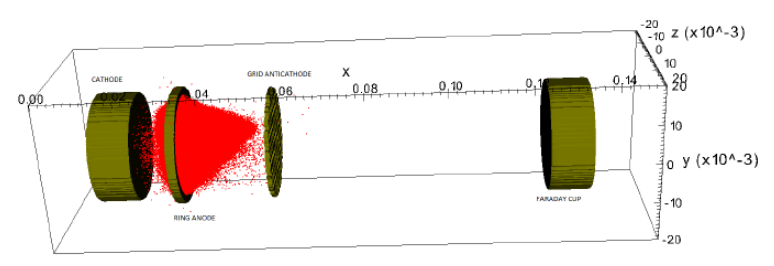

Figure 2. The example of an electron density computation (3D) in the "Vorpal" medium in the ring anode case

the main distinctions of these researches from earlier described. The experimental dependencies of this induction on the cell length and their approximations by two Gauss curves with parameters calculated by "Genfit" function of Mathcad15 are shown in Fig. 3 These approximation curves were used to recover the full magnetic field dependences upon coordinates as series expansions [10]. By the same way, that is the Laplace equation solution as series expansion the electric field distribution in the cell was found. Such distribution exists before the discharge ignition and even later - as long as it burns in Townsend mode [2, that the field is not strongly changed by plasma charges. The field distributions were indispensable to compute the charge's trajectories subject to the deceleration on the neutrals [3]. The trajectories knowledge allows one to compute the working gas ionization probability at the concrete pressure provided that the ionization cross-sections or the ionization coefficient dependences upon electron energy are known. Taken from [3] experimental dependencies for different gases were approximated by suitable functions (e.g. exponents and parabolas). By this means the full ionization probabilities during certain time for different trajectories were computed for different cell configurations and anode potentials. The comparison with the uniform magnetic field computation was made in doing so. Its induction was set either equal to the experimental axial component of the longest cell, averaged upon the cell axe length, or the induction modulus averaged upon the whole cell volume. The calculated values were compared in order to determine the utility of the different magnetic field configurations use.

The one-particle Mathcad computations show that nonuniform magnetic field is advantageous only at relatively low anode potentials. In such an event trajectories are much longer, electrons drift in the cell areas where their energies are lower, and therefore the ionization coefficient is higher. At higher anode voltages the plane of maximum ionization coefficient shifts toward cathode, where the magnetic field radial component is significant. That's why the probability of the electron hitting anode or cell well, where it recombines, sharply rises. Consequently the efficiency of this cell configuration decreases. Accounting the secondary ion-electron emission coefficient ( $\gamma$ - processes) and its energy dependence doesn't change sufficiently the whole image, though signifi- 

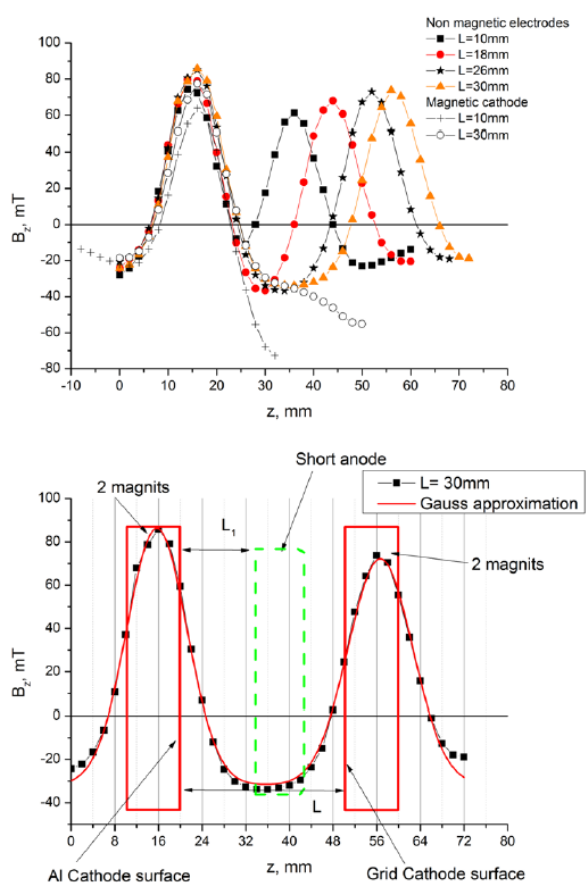

Figure 3. The experimentally determined and computed (approximating) magnetic field axial component along the cell axe

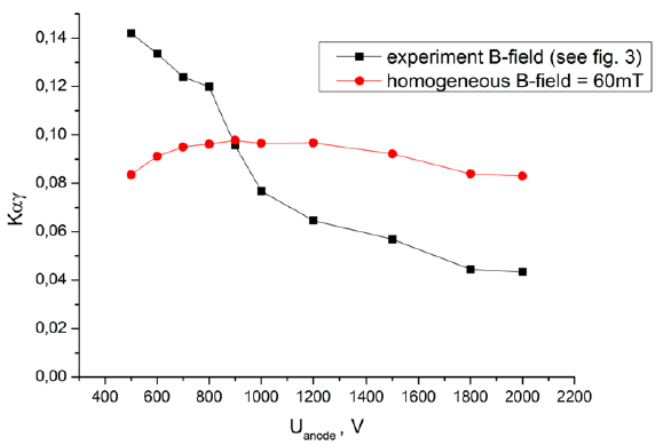

Figure 4. The combined criteria dependencies on the anode voltage in the case of a uniform and nonuniform magnetic fields

cantly changes the discharge evolution dynamics. The magnetic mirrors configuration leads to relative rising of $\alpha$ - processes role, decreasing the cathodes materials influence, aside from their magnetic properties. As an example of such one-particle computations the combined efficiency criterions dependencies upon the anode voltage are shown in Fig. 4.

The criterion is defined as the product of the ionization coefficient $\alpha(t)$, the secondary ion-electron emission coefficient $\gamma(t)$ and the mean ionization probability at the plane from which electron starts to move, integrated along the trajectory length during the definite time (in fig. 4- $35 \mathrm{~ns}$ ). The electron trajectories starting from the plane detached from cathode by 2 $\mathrm{mm}$ and from the cell axe by $1 / 5,1 / 2$ and $4 / 5$ of the cathode radius were computed. The computation

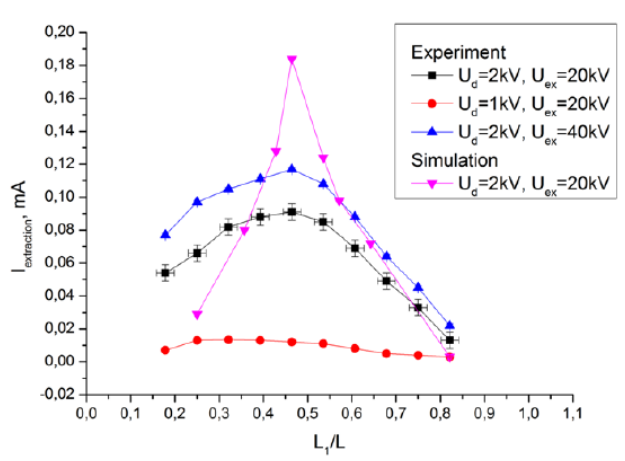

Figure 5. The experimental and computed dependencies of the extracted current on the anode location at the gas pressure $P=2 \mathrm{mTorr}$

results for the ring anode located at the $1 / 3$ cell length from cathode are shown (Fig. 4). The cell relative length is equal to 2 , whereas the uniform magnetic field induction is chosen as the simple mean of the two differently averaged magnitudes, which is $60 \mathrm{mT}$. The calculated data were summarized with the corresponding weight (the distance from the axe) simulating the integration upon the plane. The $\alpha$ and $\gamma$ magnitudes at each point were determined by the electron kinetic energy at this point. The $\gamma(E)$ dependence was taken for the hydrogen molecule ion hitting nickel surface: $\gamma(E)=0.09+1.18 \times 10^{-4} \mathrm{E}$, where E was measured in $\mathrm{eV}$. It can be seen graphically in Fig. 4 that the combined criterion for the nonuniform field is really greater at low anode voltages, whereas at voltages greater than $900 \mathrm{~V}$ it sharply decreases.

\section{The results and discussion}

The experimental and calculated dependencies of the extracted current on the ring anode arrangement in the long cell $\left(\mathrm{L} \sim \mathrm{D}_{a}\right)$ are shown in Fig. 5. A small asymmetry is seen and the maximal extracted current corresponds to $\mathrm{L}_{1} / \mathrm{L}=0.46$, that is the anode is shifted from the center toward cathode by some $\mathrm{mm}$. The sharp calculated maximum is only qualitatively proved by the experimental results - the maximums abscissas coincidence. The inconsistence between experiment and simulation points may be explained by inconsistence of the boundary conditions in the simulations. In case of the short anode the variation of its location from $\mathrm{L}_{1} / \mathrm{L}=0.07$ to 0.36 practically doesn't change the extracted current, while the discharge current changes lead to the energy efficiency (the ratio of the extracted current to the discharge power consumption) variation.

The discharge photos at different ring anode locations as well as 2D computed electron and ion distributions obtained with the Vorpal code are given in Fig. 6. The discharge contraction to the cell axe when moving the anode from the cell center is seen. The good agreement between the calculated electron density distribution in the discharge and the glow areas on 


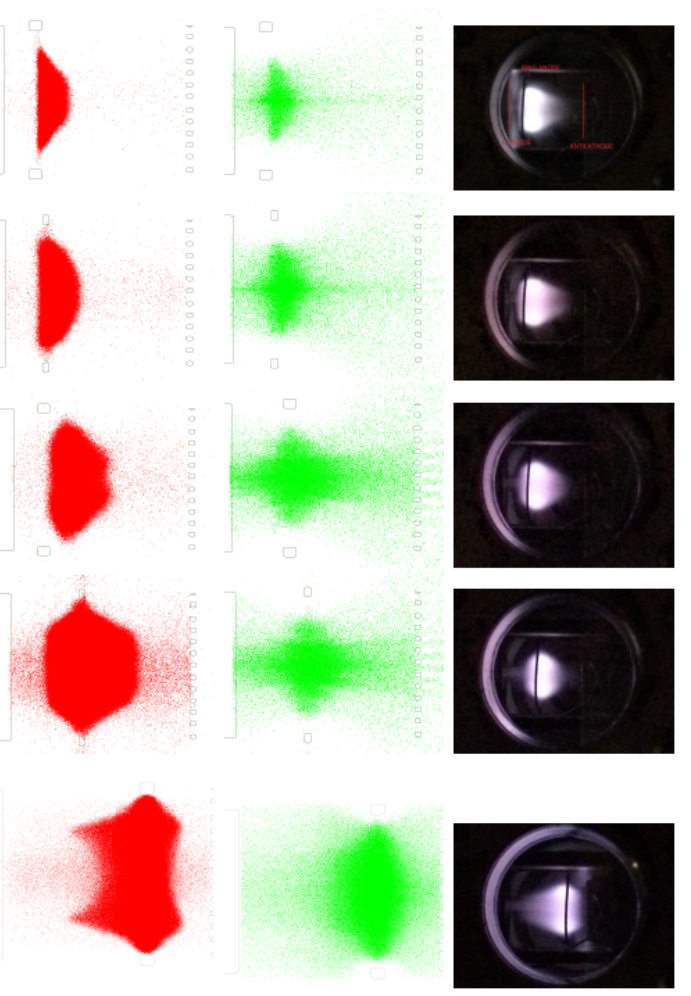

Figure 6. The discharge photos for different anodecathode distances (at the right); the computed electron (at the left) and ion densities: $L_{1} / L=0.18 ; 0.25 ; 0.36$; $0.46 ; 0.82$

the photos is seen as well. At the same time the bright "tails" of the discharge don't correlate with the ion nor electron densities in the cell volume. The "idle" discharges ignition at the external cathode's edges is clearly seen on the end photos, thus explaining the power consumption growth and extracted current fall. Under the pressure rise the external glow area shape transforms into cone, its intensity increases in the center, near axe plasma glow becomes more pronounced. At the asymmetric electrodes arrangement some discharge contraction toward the axe and elongation toward the anti-cathode takes place, so leading to the extracted current growth.

The discharge current depends on the gas pressure almost linearly for all anode forms and arrangements. It strictly follows from estimating calculations of the ionization probability linearly depending on the pressure. In the case of the ring anode (see Fig. 6, 7) the area of maximal glowing is always shifted to the center of the discharge relative to anode. It rather coincides with the calculated potential maximum area, which doesn't coincide with the anode positions marked by dips on the luminosity curves processed in ImageJ code. Besides, the magnetic induction of the nonuniform field is minimal in the center of the cell.

In the case of short and long anodes the discharge glow is uniform, as one would expect, but also maximal in the center and more pronounced near the axe (see Fig. 8 - "long" anode). It (obviously) rises under

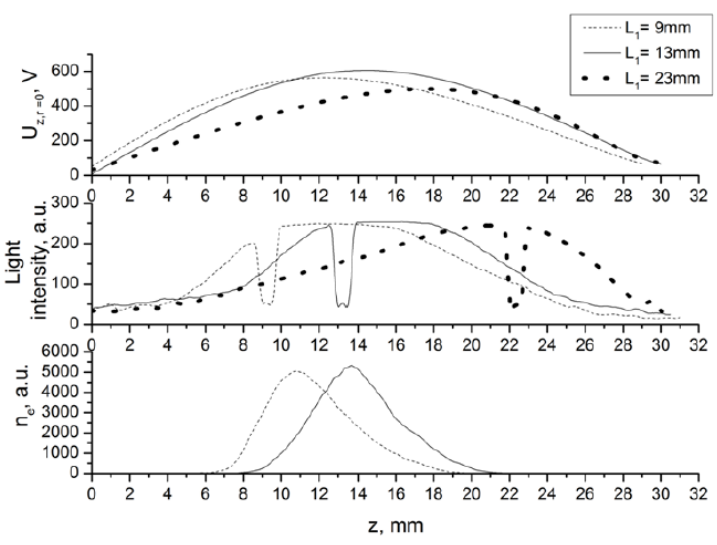

Figure 7. The electric field potential distribution (on top), a discharge glow intensity (at the center) and the computed electron density along the cell axe for different ring anode locations $\left(P=2 m\right.$ Torr, $U_{\text {discharge }}$ $\left.=2 \mathrm{kV}, U_{\text {extraction }}=20 \mathrm{kV}\right)$

pressure growth and the extracted current is higher in the whole pressure range than ring anode's one. Under pressures $\mathrm{P}>4$ mTorr the "idle" discharge ignition can be seen (glow at the right side of the anti-cathode on the right photo) which leads to sharp rise of the power consumption and consequent IS breakdowns.

As can be seen from the generalized dependencies of the extracted current $\mathrm{I}_{\text {extraction }}$, discharge current $\mathrm{I}_{\text {discharge }}$ on the discharge voltage (see Fig. 9- long anode) the highest extracted current cell is the long anode's one $\mathrm{h}_{a} / \mathrm{D}_{a} \sim \mathrm{L} / \mathrm{h}_{a} \sim 1$. In this case, as obtained in experiments, in the pressure range $\mathrm{p} \sim 0.5-2$ mTorr, discharge voltage $\mathrm{U}_{\text {discharge }} \sim 1-2 \mathrm{kV}$ and $\mathrm{U}_{\text {extraction }}=$ $20 \mathrm{kV}$ the extracted current $\mathrm{I}_{v} \sim 200-300 \mu \mathrm{A}$. In the case of the short anode, shifted to the center $\left(\mathrm{h}_{a} / \mathrm{D}_{a}\right.$ $\sim 0.5) \mathrm{I}_{\text {extraction }} \sim 100-150 \mu \mathrm{A}$ at the same anode voltage and gas pressure. The discharge ignition potential decrease from 700 to $300 \mathrm{~V}$ under the anode height rise should also be noted.

\section{Conclusion}

The preliminary results of the Penning discharge modelling are described. The specially designed test bench and open-end simulation code allow one to find out the most important laws of discharge processes and compare them with the simplified estimates made on the up to date theoretical models. The reflective discharge was visualized within the whole cell volume; its shape and structure were recorded for different anode heights and locations. The experimental proof of the discharge contraction to the cell's axe at definite combinations of geometrical (anode height and location) and physical (gas pressure and anode voltage) parameters is obtained. The good agreement of the computed electron density and the discharge glow area on photos is shown. The best cell configurations with model electrodes are found: the one providing the highest ion extraction current (long an- 


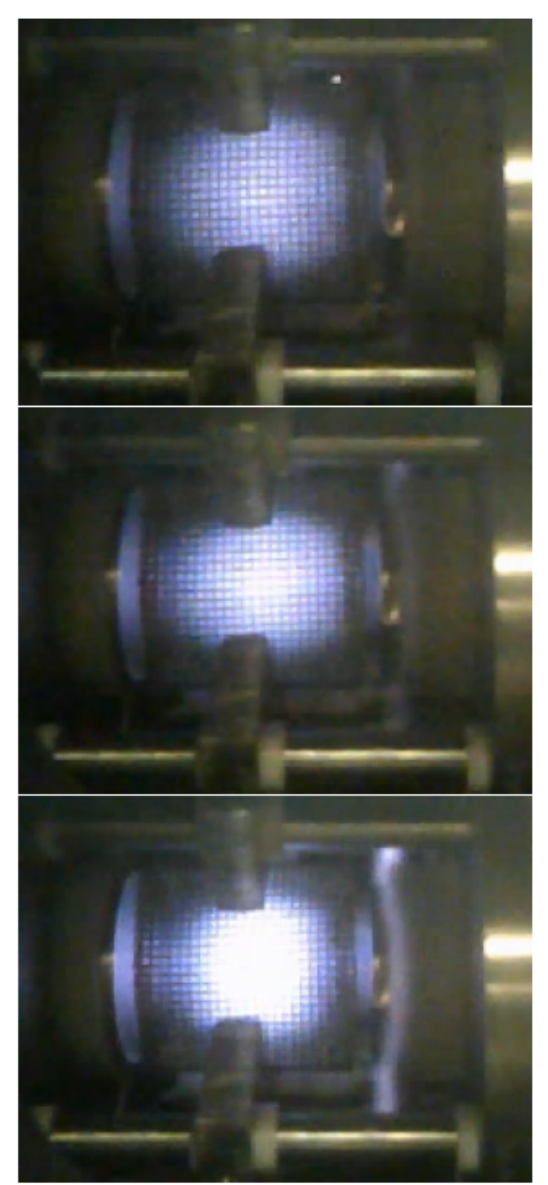

Figure 8. The discharge photos in the case of the long anode at different pressures $P=0.5 ; 1 ; 4$ mTorr $\left(U_{\text {discharge }}=2 \mathrm{kV}, U_{\text {extraction }}=20 \mathrm{kV}\right)$

ode $\mathrm{h}_{a} / \mathrm{D}_{a} \sim \mathrm{L} / \mathrm{h}_{a} \sim 1$ ) and the second providing the highest energy efficiency (short anode $\mathrm{h}_{a} / \mathrm{D}_{a} \sim 0.5$ shifted to the cathode. The one-particle computation has shown that the magnetic field nonuniformity significantly affects the discharge ignition and combustion potentials as well as the relative role of the voluminal and surface processes.

\section{References}

[1] F. M. Penning, Physica , Amsterdam, Vol. 4, 1937, p.71.

[2] W. Schururman, Physica , Amsterdam, Vol. 36, 1967, p.136.

[3] Y. Raizer, Physics of gas discharge. - Moscow, Nauka, 1987, p.591 (in Russian)

[4] I. G. Brown, The Physics and Thechology of Ion Source, J.Wiley Sons, Inc., 1989.

[5] N. V. Mamedov, N. N. Schitov, I. A. Kanshin, Physical-Chemical Kinetics in Gas Dynamics V16 (4) Http://chemphys.edu.ru/issues/2015-164/articles/590/ (2015).

[6] C. Nieter, J. R. Cary. Journal of Computational Physics 196, 448 (2004).
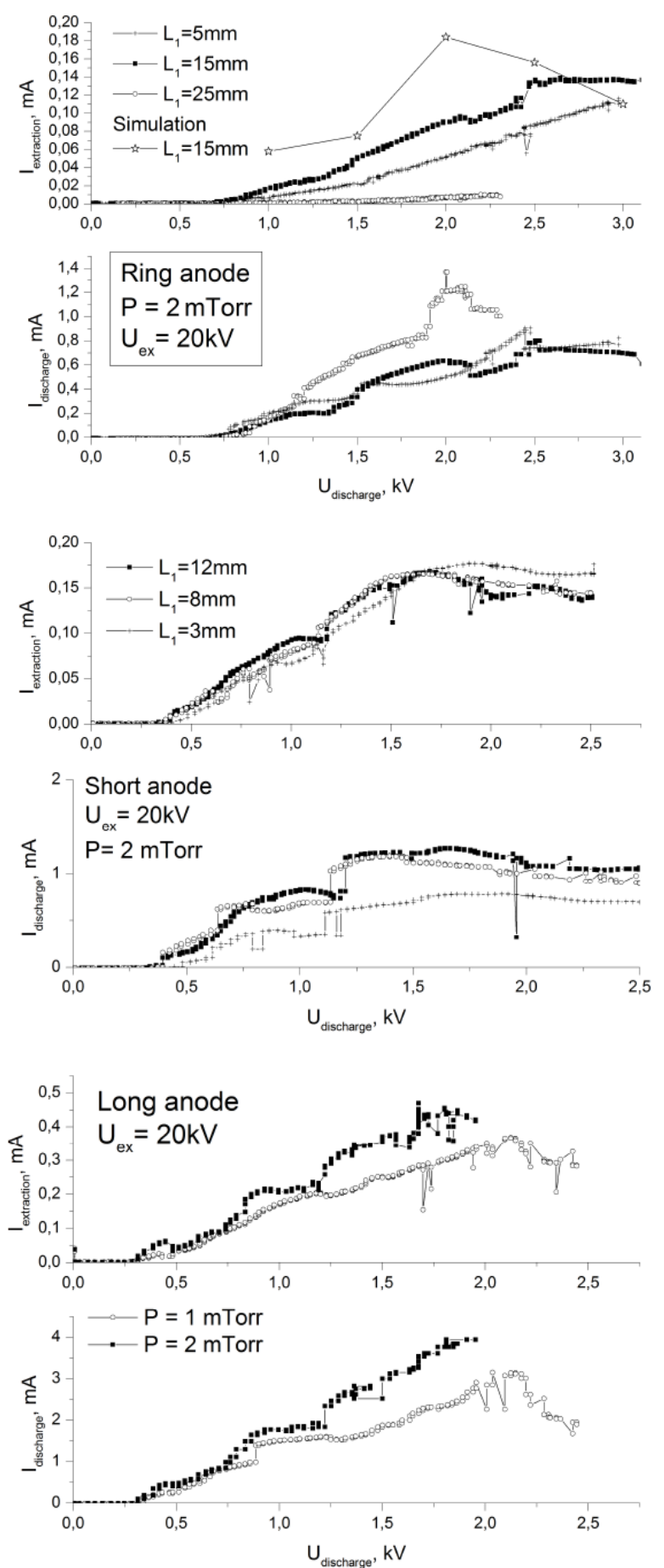

Figure 9. The dependencies of $I_{\text {extraction }}, I_{\text {discharge }}$ on the anode voltage for the ring, short and long anodes

[7] Charles K. Birdsall, A. Bruce. "Langdon", Plasma Physics via Computer, Mc Graw-Hill, Inc., New York, NY (1985)

[8] Y.Saad, Iterative methods for sparse linear systems, The PWS Publishing Company, Boston (1996)

[9] M. A. Furman, M. Pivi., Phys. Rev., ST Accel. Beams 5, 124404, 2002

[10] V. Kelman, S. Yavor, Electron optics, Leningrad, Nauka, 1968, p.487 (in Russian) 